\title{
Theoretical study on electronic states of carbon monofluoride and on the predissociation of the lower lying states
}

\author{
loannis D. Petsalakis \\ Theoretical and Physical Chemistry Institute, The National Hellenic Research Foundation, \\ 48 Vassileos Constantinou Avenue, Athens 11635 , Greece
}

(Received 24 December 1998; accepted 17 March 1999)

\begin{abstract}
Adiabatic potential energy curves of $X^{2} \Pi, 12$ doublet excited electronic states of $\mathrm{CF}$ and the ground state of $\mathrm{CF}^{+}$have been determined by MRDCI (multi reference single and double excitations configuration interaction) calculations. The potentials of the excited electronic states are complicated by interactions with repulsive states as well as by valence-Rydberg interactions. Potential energy curves of higher-lying Rydberg states have been generated with the aid of quantum defect calculations. Radiative lifetimes have been calculated for the $A^{2} \Sigma^{+}$and the $B^{2} \Delta$ states while predissociation lifetimes have been obtained by a five-state complex-coordinate rotation calculation involving the lowest five doublet states along with their mutual interactions. The results are in agreement with the existing experimental data regarding the predissociation of the $v>1$ levels of $A^{2} \Sigma^{+}$, while predissociation is predicted for all the vibrational-rotational levels of the $2^{2} \Sigma^{+}$, for the $v>1$ levels of the positive parity substate of $D^{2} \Pi$ and for the higher vibrational levels $(v$ $>2$ ) of $B^{2} \Delta$. For the latter two states predissociation increases significantly with the rotational level. (C) 1999 American Institute of Physics. [S0021-9606(99)30822-9]
\end{abstract}

\section{INTRODUCTION}

The spectroscopy of the CF radical has been studied by many different methods (Refs. 1-6 and references therein). Two band systems of the doublet manifold, $A^{2} \Sigma^{+}-X^{2} \Pi$ in the region $2075-3000 \AA$ and $B^{2} \Delta-X^{2} \Pi$ in $1900-2200 \AA$, and the $a^{4} \Sigma^{-}-X^{2} \Pi$ transition have been identified and characterized, while a number of new bands in the region 7.3 to $7.89 \mathrm{eV}$, associated with higher electronic states have been obtained but have not been well characterized. ${ }^{6}$ The $A^{2} \Sigma^{+}$ state has been found to be predissociated above $v=1$ (Ref. 3) and several mechanisms for the predissociation have been postulated, including interaction with a ${ }^{4} \Pi$ state, ${ }^{3}$ and indirect interaction with a ${ }^{4} \Sigma^{-}$state. ${ }^{6}$ In recent laser-induced fluorescence work on the $A^{2} \Sigma^{+}-X^{2} \Pi$ transition, ${ }^{5}$ the lifetimes of vibrational levels of the $A^{2} \Sigma^{+}$state are found to be in agreement with the results of a theoretical calculation, ${ }^{7}$ where the predissociation involves tunneling through a barrier in the potential energy curve of the $A^{2} \Sigma^{+}$state which is presumed to result from an avoided crossing with another ${ }^{2} \Sigma^{+}$state at large internuclear distances.

Except for the above-mentioned $a b$ initio study which involved only the $X^{2} \Pi, A^{2} \Sigma^{+}$, and $B^{2} \Delta$ states, earlier HF and MCHF calculations on the electronic states of CF have yielded information on several valence and Rydberg states of this system. ${ }^{6}$ The potential energy curves thus obtained show crossings at large internuclear distances $(R)$ between valence states with different dissociation limits and at short $R$ crossings with Rydberg states. For example, the $B^{2} \Delta$ state is predicted to have an avoided crossing with a Rydberg $3 d^{2} \Delta$ state at short $R$ (which was not included in the latest theoretical work on this state). ${ }^{7}$ However, in these HF and MCHF calculations it was not possible to obtain the different types of states (or different parts of a potential energy curve) with one set of calculations and thus to obtain them with similar accuracy ${ }^{6}$ although they did yield a lot of information on the configurations characterizing the different states in the different regions of the internuclear distance.

Firstly, in the present work large-scale multireference configuration interaction calculations have been carried out on the ground and doublet excited states of CF in an effort to obtain uniform-accuracy potential energy curves for the excited states of $\mathrm{CF}$ including the higher states which might be associated with the observed and as yet unassigned bands. Secondly, quantum defect functions have been determined for Rydberg $s, p$, and $d$ states which may be used to generate higher $n s, n p$, and $n d$ Rydberg states. Thirdly, transition dipole moments, radial coupling matrix elements $d / d R$, and rotational-electronic coupling matrix elements connecting the states $X^{2} \Pi, A^{2} \Sigma^{+}, B^{2} \Delta, 2^{2} \Pi$, and $2^{2} \Sigma^{+}$have been calculated. The potential energy curves and the interaction matrix elements of these five states have been included in a five-state complex-coordinate-scaling calculation of their vibrational-rotational levels and widths. The object of the latter calculations was to determine predissociation lifetimes for vibrational levels of the above excited states in a multistate treatment, including all the possible interactions between them.

\section{CALCULATIONS}

\section{A. Ab initio calculations}

$A b$ initio $\mathrm{MRDCI}^{8}$ calculations have been carried out on the doublet electronic states of CF for 16 values of the internuclear distance ranging from 1.7 to 4.5 bohr. Similar calculations have been carried out on electronic states of the cation, $\mathrm{CF}^{+}$, of which only the ground-state potential is required for the present work. The $\mathrm{AO}$ basis set employed 
consists of the (10s6p/5s4p) sets for $\mathrm{C}$ and $\mathrm{F}$ of Dunning 9 augmented with $d$ polarization functions with exponent 0.75 for $\mathrm{C}$ and 0.90 for $\mathrm{F}$, and with Rydberg functions $2 s$ and $2 p$ for $\mathrm{F}$ and $2 s, 2 p$, and $2 d$ functions for $\mathrm{C}$, with exponents as in Dunning and Hay. ${ }^{10}$

The calculations have employed $C_{2 v}$ point group symmetry where the ${ }^{2} \Sigma^{+}$states correspond to ${ }^{2} A_{1}$ states, the ${ }^{2} \Delta$ to ${ }^{2} A_{1}$ and ${ }^{2} A_{2}$, the ${ }^{2} \Sigma^{-}$to ${ }^{2} A_{2}$, and the ${ }^{2} \Pi$ to ${ }^{2} B_{1}$ and ${ }^{2} B_{2}$. Five roots of each symmetry, ${ }^{2} A_{1},{ }^{2} A_{2}$, and ${ }^{2} B_{1}$ have been calculated at each value of the internuclear geometry. In order to have a uniform description of the electronic states over $R$, rather large sets of reference configurations, determined on the basis of preliminary calculations at different values of the internuclear distance, have been employed. For the ${ }^{2} A_{1}$ calculations the reference space consisted of 65 reference configurations (yielding 115 configuration functions), for the ${ }^{2} B_{1}$ calculations there were 68 reference configurations (135 configuration functions), and for the ${ }^{2} A_{2}$ calculations there were 68 reference configurations (187 configuration functions). For the ${ }^{1} A_{1}$ states of $\mathrm{CF}^{+}$there were 72 reference configurations ( 89 configuration functions). From the sets of all the configurations generated by all single and double excitations with respect to the reference functions, selection was carried with a threshold of $1 \times 10^{-6}$ hartree and the resulting CI spaces were of size between 40000 and 73000 , for different values of $R$. Extrapolation to zero threshold and the full CI correction ${ }^{11}$ was applied to the eigenvalues.

For the $X^{2} \Pi, A^{2} \Sigma^{+}, B^{2} \Delta, 2^{2} \Pi$, and $2^{2} \Sigma^{+}$states, dipole transition moments and interaction matrix elements over the electronic wavefunctions have been calculated for all the above values of the internuclear distance $R$. The interaction matrix elements are radial coupling for pairs of states with the same $\Lambda$, for which the matrix elements of $d / d R$ have been calculated and the rotational-electronic coupling matrix elements for pairs of states differing in $\Lambda$ by 1 .

Some additional MRDCI calculations on the electronic states of CF have been carried out, one set employing only single-configuration (valence and Rydberg) reference spaces as an aid to understanding the shape of the potential energy curves, and one set of calculations employing reference spaces consisting of only single open-shell configurations, in order to separate the Rydberg contributions from the valence contributions. The potential energy curves obtained from the above calculations on the Rydberg states, $E(R)$, along with the potential energy curve of the ground state of $\mathrm{CF}^{+}$, $V_{i}^{+}(R)$, have been employed to calculate quantum defect functions $\eta_{i l \lambda}(R)$ where $i$ refers to the ionic state and $l \lambda$ to the particular channel, ${ }^{12,13}$ from

$$
\tan \pi \eta_{i}(R)+\frac{\tan \pi \nu_{i}(R)}{A_{l_{i}}\left(\nu_{i}(R)\right)}=0,
$$

where

$$
\nu_{i}(R)=\left[2\left(V_{i}^{+}(R)-E(R)\right)\right]^{-1 / 2},
$$

and

$$
A_{l_{i}}\left(\nu_{i}(R)\right)=\prod_{k=0}^{l_{i}}\left(1-\frac{k^{2}}{\nu_{i}^{2}}\right) .
$$

The factor $\left[A_{l_{i}}\left(\nu_{i}(R)\right)\right]^{-1}$ serves to eliminate solutions with $l_{i}<n_{i} \cdot{ }^{13}$ Equation (1) is employed first, with the ab initio potentials substituted for $E(R)$, for the determination of the quantum defects. The higher Rydberg states are calculated subsequently by introducing the calculated quantum defect functions in Eq. (1) and varying $E(R)$ to find those energies which satisfy the equation. In this manner all the $l \lambda$ Rydberg states up to the ionization limit are generated.

\section{B. Multistate vibrational calculations involving complex scaling of the internuclear coordinate}

A multistate-complex scaling method for the calculation of predissociation resonances that has been described in detail elsewhere ${ }^{12,14}$ has been employed for the present fivestate $\left(X^{2} \Pi^{+}, A^{2} \Sigma^{+}, B^{2} \Delta^{+}, 2^{2} \Pi^{+}\right.$, and $\left.2^{2} \Sigma^{+}\right)$calculations of vibrational rotational levels and their widths.

The transformation of the nuclear coordinate $R \rightarrow \rho$ $=R e^{i \theta}$ with $\theta$ real, allows the calculation of energies and widths of predissociation resonances in terms of square integrable functions by solution of a complex eigenvalue problem

$$
|\underline{\underline{H}}-\underline{\underline{z}}|=0,
$$

where $\underline{\underline{z}}$ is the diagonal matrix of complex eigenvalues. The resonances correspond to the eigenvalues $z_{k}$ which are stable with respect to $\theta$ and

$$
z_{k}=E_{k}-\frac{i}{2} \Gamma_{k},
$$

where $E_{k}$ is the energy position and $\Gamma_{k}$ is the linewidth of the resonance.

The Hamiltonian matrix in Eq. (4) consists of blocks $\underline{\underline{H}}^{I J}$ where $I, J$ stand for the different electronic states. A basis set of Hermite polynomials is employed and matrix elements over the nuclear energy operator and over the electronic potential energy functions and interactions are computed, where the latter are evaluated numerically with the aid of a Gauss-Hermite quadrature procedure. ${ }^{12}$

In the present case we use 100 harmonic oscillator functions per state which result in a $500 \times 500$ complex eigenvalue problem. Stabilization of the eigenvalues was achieved for complex rotation angles from $2^{\circ}$ to $10^{\circ}$ and for some of the eigenvalues for larger values of $\theta$.

\section{RESULTS AND DISCUSSION}

\section{A. Potential energy curves}

The $a b$ initio total energies calculated in the present work for electronic states of CF and the ground state of $\mathrm{CF}^{+}$ have been listed in Table I. Potential energy curves have been plotted in Fig. 1 for the ${ }^{2} \Sigma^{+}$and the ${ }^{2} \Pi$ states and in Fig. 2 for the ${ }^{2} \Delta$ and the ${ }^{2} \Sigma^{-}$states, showing the expected complicated shapes, which result from their mutual interactions and avoided crossings. The potential energy curve of the ground state of the cation, $\mathrm{CF}^{+}$has also been plotted in both figures as a reference for the potentials with Rydberg character. As shown in Fig. 1, the potential energy curves of all the excited ${ }^{2} \Pi$ states calculated in the present work have 
TABLE I. Calculated total energies (hartree) $)^{\mathrm{a}}$ of electronic states of $\mathrm{CF}$ and the ground state of $\mathrm{CF}^{+}$.

\begin{tabular}{|c|c|c|c|c|c|c|c|}
\hline$R$ & $A^{2} \Sigma^{+}$ & $2{ }^{2} \Sigma^{+}$ & $3^{2} \Sigma^{+}$ & $1^{2} \Delta$ & $2^{2} \Delta$ & $3^{2} \Delta$ & $2^{\prime 2} \Delta$ \\
\hline 1.7 & -1.089741 & -1.039905 & -1.010359 & -1.002038 & & & \\
\hline 1.8 & -1.194641 & -1.146150 & -1.115454 & -1.107200 & -1.108398 & -1.081285 & \\
\hline 2.0 & -1.297735 & -1.254438 & -1.223109 & -1.213692 & -1.214273 & -1.187682 & \\
\hline 2.2 & -1.322304 & -1.283116 & -1.249123 & -1.241401 & -1.240781 & -1.213363 & \\
\hline 2.4 & -1.310154 & -1.280176 & -1.240198 & -1.279778 & -1.227475 & -1.198642 & \\
\hline 2.6 & -1.291565 & -1.272346 & -1.238707 & -1.284357 & -1.198136 & -1.168262 & \\
\hline 2.8 & -1.306560 & -1.246811 & -1.231010 & -1.277064 & -1.165685 & -1.133780 & \\
\hline 3.0 & -1.324448 & -1.232395 & -1.207128 & -1.268792 & -1.132129 & -1.104892 & \\
\hline 3.2 & -1.335458 & -1.228675 & -1.178001 & -1.261803 & -1.100235 & -1.071237 & -1.195702 \\
\hline 3.4 & -1.338520 & -1.229255 & -1.208911 & -1.258502 & -1.071342 & -1.041641 & -1.231302 \\
\hline 3.6 & -1.336654 & -1.240126 & -1.226557 & -1.258735 & -1.047470 & -1.018174 & -1.255297 \\
\hline 3.8 & -1.333138 & -1.256540 & -1.234273 & -1.274548 & -1.026830 & -0.995239 & -1.261016 \\
\hline 4.0 & -1.328688 & -1.270197 & -1.241506 & -1.288820 & -1.010559 & -0.978591 & -1.263272 \\
\hline 4.2 & -1.324612 & -1.278127 & -1.248590 & -1.298327 & & & -1.265562 \\
\hline 4.4 & -1.321718 & -1.282314 & -1.254999 & -1.305951 & & & -1.266951 \\
\hline \multirow[t]{2}{*}{4.5} & -1.321405 & -1.283180 & -1.258190 & -1.309470 & -0.983812 & -0.967338 & -1.268955 \\
\hline & $X^{2} \Pi$ & $D^{2} \Pi$ & $3^{2} \Pi$ & $4{ }^{2} \Pi$ & $5^{2} \Pi$ & $1^{2} \Sigma^{-}$ & $1{ }^{1} \Sigma^{+}$ \\
\hline 1.7 & -1.156090 & -1.044012 & -1.002215 & -0.994674 & -0.979339 & & -0.952681 \\
\hline 1.8 & -1.285790 & -1.150273 & -1.107880 & -1.100378 & -1.084393 & -0.926417 & -1.058446 \\
\hline 2.0 & -1.434899 & -1.255776 & -1.214632 & -1.206272 & -1.189129 & -1.090684 & -1.165719 \\
\hline 2.2 & -1.497472 & -1.280757 & -1.240912 & -1.231715 & -1.214541 & -1.178234 & -1.193384 \\
\hline 2.4 & -1.514922 & -1.267670 & -1.227438 & -1.218811 & -1.202401 & -1.220959 & -1.182142 \\
\hline 2.6 & -1.509765 & -1.238357 & -1.197306 & -1.188759 & -1.171325 & -1.242168 & -1.153236 \\
\hline 2.8 & -1.498991 & -1.233062 & -1.203608 & -1.190011 & -1.164915 & -1.255088 & -1.120113 \\
\hline 3.0 & -1.481933 & -1.258171 & -1.216669 & -1.209902 & -1.168733 & -1.270085 & -1.086404 \\
\hline 3.2 & -1.462343 & -1.275851 & -1.233064 & -1.227529 & -1.179804 & -1.279122 & -1.055019 \\
\hline 3.4 & -1.441966 & -1.290151 & -1.247747 & -1.237683 & -1.206498 & -1.289272 & -1.027094 \\
\hline 3.6 & -1.422481 & -1.300337 & -1.256144 & -1.246248 & -1.226315 & -1.299752 & -1.002859 \\
\hline 3.8 & -1.404773 & -1.307943 & -1.263526 & -1.252073 & -1.239545 & -1.306230 & -0.983559 \\
\hline 4.0 & -1.389086 & -1.313446 & -1.270258 & -1.257242 & -1.249244 & -1.310601 & -0.968312 \\
\hline 4.2 & -1.375626 & -1.316842 & -1.273821 & -1.261063 & -1.255639 & -1.315942 & -0.954871 \\
\hline 4.4 & -1.363888 & -1.320356 & -1.276999 & -1.264585 & -1.259288 & -1.319042 & -0.946206 \\
\hline 4.5 & -1.358476 & -1.321208 & -1.277023 & -1.265791 & -1.260968 & -1.320516 & -0.942261 \\
\hline
\end{tabular}

a -136.000000 should be added to the entries.

characteristic Rydberg shapes at short $R$ while for $R$ about 2.6 bohr they have maxima which result from avoided crossings with repulsive valence states.

At large $R$ the ${ }^{2} A_{1}$ calculations have yielded three ${ }^{2} \Sigma^{+}$ states and two ${ }^{2} \Delta$, while at short $R$ at some points a fourth ${ }^{2} \Sigma^{+}$is obtained and at others a second Rydberg ${ }^{2} \Delta$. The potential energy curves of the two higher ${ }^{2} \Sigma^{+}$states are

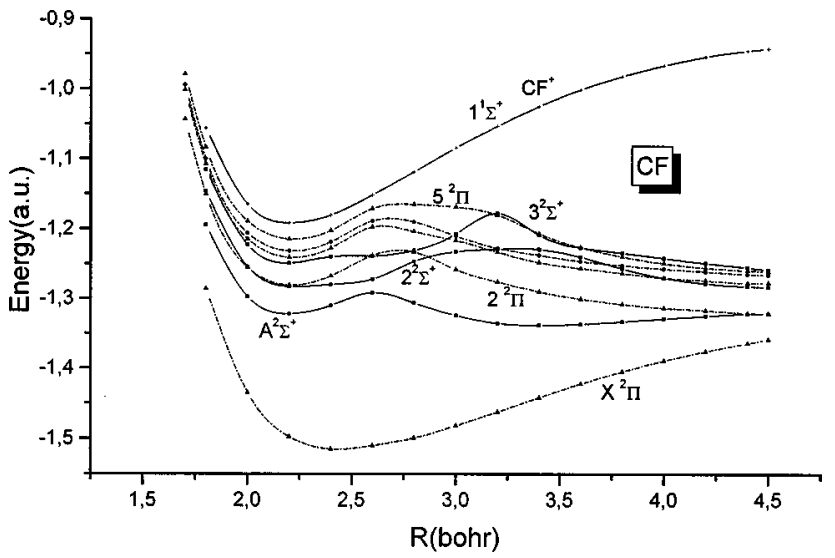

FIG. 1. Potential energy curves of ${ }^{2} \Pi$ (dot-dashed lines) and ${ }^{2} \Sigma^{+}$(solid lines) electronic states of $\mathrm{CF}$ and the ground state of $\mathrm{CF}^{+}$. rather peculiar at short $R$. However their shape is the result of avoided crossings as may be seen in Fig. 3, where "diabatic" states, obtained from single-reference calculations corresponding to the important configurations of the ${ }^{2} \Sigma^{+}$states at different $R$, have been plotted along with the multireference ${ }^{2} \Sigma^{+}$states. In particular, the lowest ${ }^{2} \Sigma^{+}$(i.e., the $A$ )

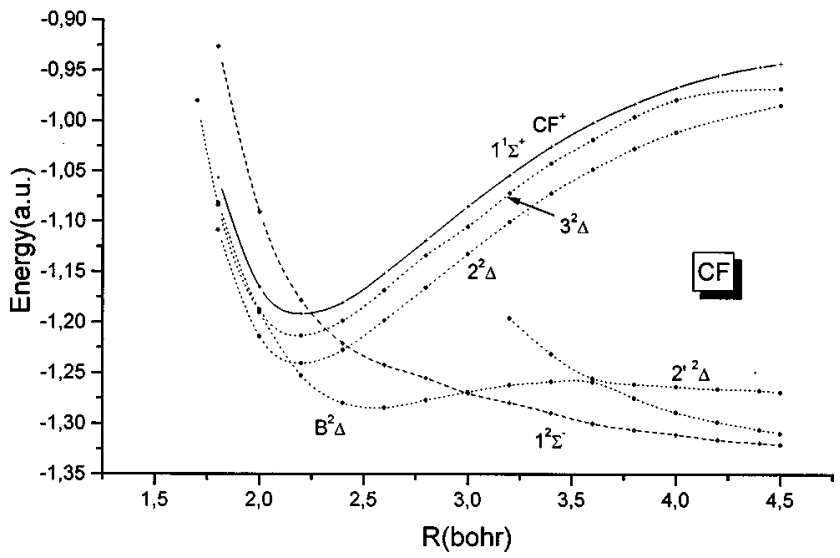

FIG. 2. Potential energy curves of ${ }^{2} \Delta$ (dotted lines) and ${ }^{2} \Sigma^{-}$(dashed line) electronic states of $\mathrm{CF}$ and the ground state of $\mathrm{CF}^{+}$. 


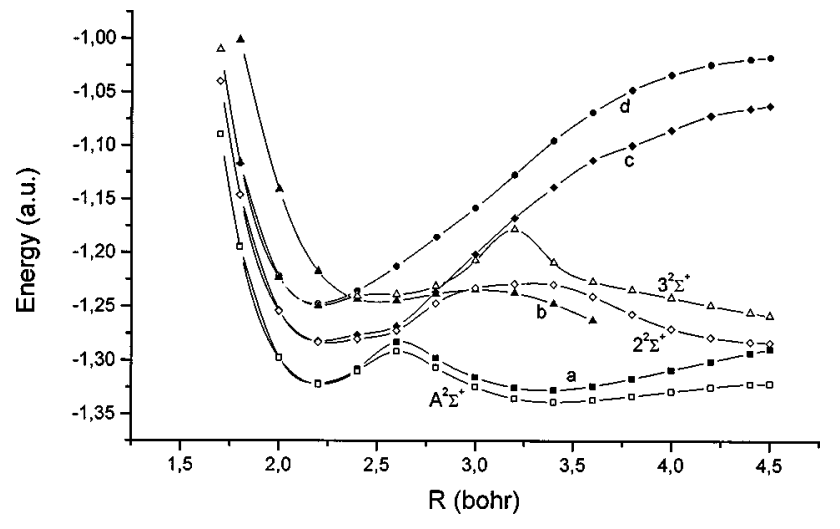

FIG. 3. Adiabatic potential energy curves for the first three ${ }^{2} \Sigma^{+}$electronic states of CF (open squares, open diamond, and open triangles, respectively) and potential energy curves of the diabatic states characterized by excitations $2 \pi \rightarrow 6 \sigma$ (curve a), $5 \sigma \rightarrow 2 \pi$ (curve b), $2 \pi \rightarrow 7 \sigma$ (curve $\mathbf{c}$ ), and $2 \pi$ $\rightarrow 8 \sigma$ (curve d) with respect to the ground state configuration (see text).

state is characterized throughout by a single configuration, with one open shell in the $6 \sigma$ orbital or a $2 \pi \rightarrow 6 \sigma$ excitation with respect to the ground state configuration $1 \sigma^{2} 2 \sigma^{2} 3 \sigma^{2} 4 \sigma^{2} 5 \sigma^{2} 1 \pi^{4} 2 \pi$. However, the character of the state changes since at short $R$ the $6 \sigma$ orbital has Rydberg $s$ character while at $R$ larger than 2.6, it has valence character. The Rydberg $s$ character is taken on for $R$ larger than 2.6 bohr by the next $\sigma$ orbital, which at shorter $R$ has Rydberg $p$ character. The change in the Rydberg character of the second ${ }^{2} \Sigma^{+}$state is indicated as an avoided crossing at 2.6 bohr (see Fig. 3). At large $R$ for the second ${ }^{2} \Sigma^{+}$state, starting at about $2.8 \mathrm{bohr}$, and for the region $2.6-2.8 \mathrm{bohr}$ for the third ${ }^{2} \Sigma^{+}$ state, the potential energy curves follow the $5 \sigma \rightarrow 2 \pi$ (with respect to the ground state configuration) diabatic state (solid triangles in Fig. 3), while at $R$ larger than 3.5 bohr, the configuration $5 \sigma^{2} \rightarrow 6 \sigma 2 \pi$ also becomes important.

Virtually identical energies are obtained by the ${ }^{2} A_{1}$ and ${ }^{2} A_{2}$ calculations of the ${ }^{2} \Delta$ states. As shown in Fig. 2 , the potential energy curve of $B^{2} \Delta$ shows a maximum at $3.6 \mathrm{bohr}$ which is a result of an avoided crossing with a repulsive ${ }^{2} \Delta$ state correlating with ground state dissociation limits (state $2^{\prime 2} \Delta$ in Table I). The potential energy curves of the higher ${ }^{2} \Delta$ states shown in Fig. 2, also have the characteristic Rydberg shapes with minima at $2.2 \mathrm{bohr}$, where the avoided crossing between the first two (the valence and the Rydberg) ${ }^{2} \Delta$ states has been depicted in the plot as a crossing. The valence ${ }^{2} \Delta$ state is characterized by the $5 \sigma \rightarrow 2 \pi$ configuration for $R$ out to $3.8 \mathrm{bohr}$, at which point and for larger $R$ the configuration $5 \sigma^{2} \rightarrow 6 \sigma 2 \pi$ gains importance. The ${ }^{2} \Sigma^{-}$state,
TABLE II. Vertical transition energies (eV) at $R_{\min }$ of $X^{2} \Pi$ and adiabatic

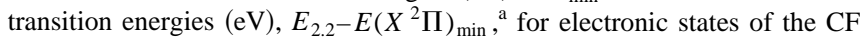
molecule.

\begin{tabular}{cccc}
\hline \hline State & Type & $\Delta E_{v}(\mathrm{eV})$ & $\Delta E_{\mathrm{ad}}(\mathrm{eV})$ \\
\hline$A^{2} \Sigma^{+}$ & $\mathrm{Rs}$ & 5.65 & $5.25(5.293)^{\mathrm{b}}$ \\
$2^{2} \Sigma^{+}$ & $\mathrm{Rp}$ & 6.40 & $6.31(6.35)^{\mathrm{c}}$ \\
$3^{2} \Sigma^{+}$ & $\mathrm{Rd}$ & 7.48 & 7.24 \\
$4^{2} \Sigma^{+}$ & $\mathrm{Rs}$ & $\ldots$ & 7.48 \\
$B^{2} \Delta$ & $\mathrm{V}$ & 6.36 & $6.27(6.125)^{\mathrm{b}}$ \\
$2^{2} \Delta$ & $\mathrm{Rd}$ & 7.88 & 7.45 \\
$3^{2} \Delta$ & $\mathrm{Rd}$ & 8.60 & 8.00 \\
$D^{2} \Pi$ & $\mathrm{Rp}$ & 6.82 & $6.38(6.481)^{\mathrm{b}}$ \\
$3^{2} \Pi$ & $\mathrm{Rd}$ & 7.91 & 7.46 \\
$4^{2} \Pi$ & $\mathrm{Rp}$ & 8.15 & 7.71 \\
$5^{2} \Pi$ & $\mathrm{Rd}$ & 8.61 & 8.18 \\
$1^{2} \Sigma^{-}$ & $\mathrm{V}$ & 7.96 & $\ldots$ \\
$1^{1} \Sigma^{+}\left(\mathrm{CF}^{+}\right)$ & & $9.06^{\mathrm{d}}$ & $8.75^{\mathrm{e}}$ \\
\hline \hline
\end{tabular}

${ }^{\mathrm{a}} R_{\min }=2.4223$ bohr, $E\left(X^{2} \Pi\right)_{\min }=-137.515069$ hartree.

${ }^{\mathrm{b}}$ Experimental $T_{e}$ (Ref. 15).

${ }^{\mathrm{c}}$ Experimental $\Delta E_{0-0}$ (Ref. 6).

${ }^{\mathrm{d}}$ Experimental vertical IP 9.23 \pm 0.08 (Ref. 16), $9.17 \pm 0.17$ (Ref. 17), 9.55 \pm 0.01 (Ref. 18).

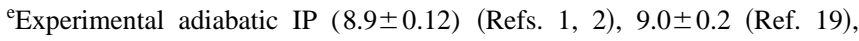
$9.11 \pm 0.01$ (Ref. 18)

correlating with ground state dissociation limits is repulsive and extends back through the other states and above the first ionization limit.

In Table II, the vertical transition energies at 2.4223 bohr (which is the minimum of the fitted potential energy curve of the ground state), and the adiabatic electronic transition energies of the excited states with respect to the ground state have been listed, while the available experimental $T_{e}$ values $(6,15)$ are also included in brackets. Also listed are the computed vertical and adiabatic ionization potentials. The labels listed next to the states in Table II refer to the Rydberg $(R)$ or valence $(V)$ character of the excited states, while the atomic $l$ labels $s, p$, or $d$ refer to the predominant contribution of the corresponding atomic orbitals with small exponents to the Rydberg state. These $l$ labels are rather formal since $l$-mixing is allowed in the molecular symmetry. As shown in Table II, the $\Delta E_{\text {adiab }}$ obtained from the potential energy minima is very close to the experimental energy difference, within $0.05 \mathrm{eV}$ for the $A^{2} \Sigma^{+}$state. For the $2^{2} \Pi(3 p)$ state (or $\left.D^{2} \Pi\right)$ the theoretical transition energy differs by $0.1 \mathrm{eV}$ from the experimental, while for the valence $B^{2} \Delta$ state the error in the theoretical transition energy is $0.13 \mathrm{eV}$. These differences of the theoretical transition energies from the experimental are typical of such calcula-

TABLE III. Expansion coefficients of the quantum defect functions [cf. Eq. (6)]. ${ }^{\mathrm{a}}$

\begin{tabular}{ccrrrrr}
\hline \hline Channel & & \multicolumn{1}{c}{$a_{0}$} & \multicolumn{1}{c}{$a_{1}$} & \multicolumn{1}{c}{$a_{2}$} & \multicolumn{1}{c}{$a_{3}$} & $a_{4}$ \\
\hline${ }^{2} \Sigma^{+}$ & $3 s$ & 11.413149 & -16.715957 & 9.989543 & -2.618930 & 0.252706 \\
${ }^{2} \Sigma^{+}$ & $3 p$ & -31.641893 & 57.733006 & -39.984122 & 12.301635 & -1.419764 \\
${ }^{2} \Pi$ & $3 p$ & 5.872032 & -9.878012 & 5.859449 & -1.553864 & 0.153471 \\
${ }^{2} \Pi$ & $3 d$ & -57.978214 & 97.728264 & -61.080230 & 16.676914 & -1.682657 \\
${ }^{2} \Delta$ & $3 d$ & 0.928098 & -0.851989 & -0.025280 & 0.105012 & -0.016945 \\
\hline \hline
\end{tabular}

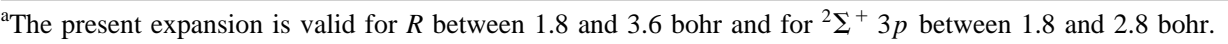




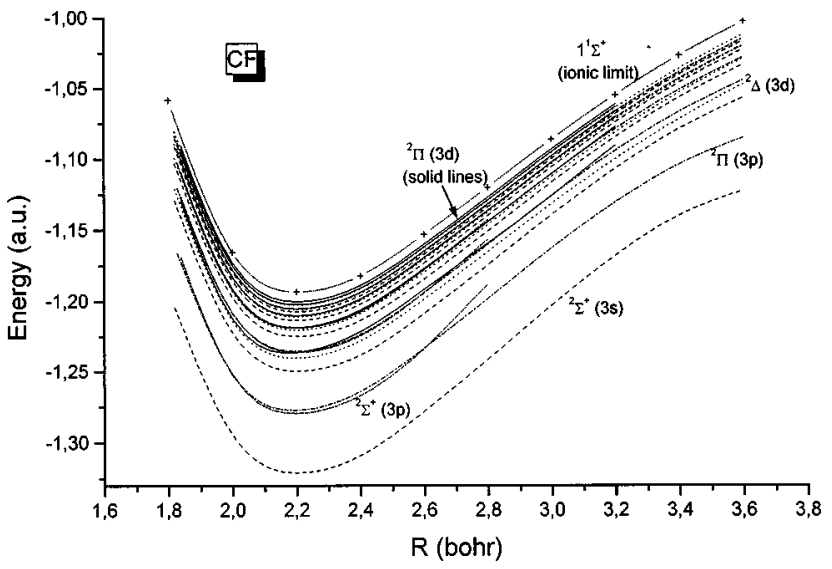

FIG. 4. Potential energy curves of $s, p$, and $d$ Rydberg states of CF, generated by the quntum defect calculations. (Dashed lines) $s^{2} \Sigma^{+}$, (dash-dot) $p^{2} \Pi$, (short dash-dot) $p^{2} \Sigma^{+}$, (dot) $d^{2} \Delta$, and (solid lines) $d^{2} \Pi$.

tions and are comparable to those of the previous CI work on the $X^{2} \Pi, A^{2} \Sigma^{+}$, and $B^{2} \Delta$ states. $^{7}$

On the basis of the transition energies listed in Table II, there is no evidence of a ${ }^{2} \Sigma^{+}$state referred to as $C^{\prime}(6,15)$ at $6.64 \mathrm{eV}$, and therefore the observed bands must be assigned to a different transition, not involving any of the Rydberg states. The assignment of the observed band at $6.35 \mathrm{eV}$ to the transition $2^{2} \Sigma^{+}(3 p)-X^{2} \Pi$ (Ref. 6) is also consistent with the calculated $\Delta E_{\text {adiab }}$ (cf. Table II). As mentioned in the introduction, additional bands in the absorption spectra of CF associated with transitions involving Rydberg states have been observed and not well characterized. ${ }^{6}$ For example there is reference to a transition called $F-X^{2} \Pi$ with $F$ either $\mathrm{a}^{2} \Sigma^{+}$or a ${ }^{2} \Delta$ state at $7.32 \mathrm{eV}$, to a transition $H^{2} \Sigma^{+}-X^{2} \Pi$ at $7.38 \mathrm{eV}$ and to a close-lying transition $G^{2} \Pi-X^{2} \Pi$, which has been tentatively associated with the $3 d$ Rydberg states. The Rydberg states of the present work which are labeled $3 d, 4{ }^{2} \Sigma^{+}$at $\Delta E_{\text {ad }} 7.48 \mathrm{eV}, 3^{2} \Pi$ at $7.46 \mathrm{eV}$, and $2^{2} \Delta$ at $7.45 \mathrm{eV}$ are within $0.12 \mathrm{eV}$ of the above experimental energies and would support the assignment of the above transitions to the $3 d$ Rydberg states and the assignment of $2^{2} \Delta(3 d)$ to the experimental $F$ state. Additional bands have been observed at 7.52, 7.60, 7.69, and $7.89 \mathrm{eV}$ some of which may be associated with transitions involving the higher Rydberg states of Table II, depending on whether the observed energies refer to vertical or adiabatic transitions. For example, the $4^{2} \Pi(4 p)$ state calculated at $\Delta E_{\text {ad }} 7.71 \mathrm{eV}$ might be involved in the $7.60 \mathrm{eV}$ band while the $3^{2} \Delta(4 d)$ at $8.00 \mathrm{eV}$ might be involved in the $7.89 \mathrm{eV}$ band.

As mentioned above the Rydberg states of CF have been calculated by multireference CI calculations where the reference spaces consisted of only single open shell configurations. In this manner it was possible to construct "diabatic" Rydberg potential energy curves, for the $3 s$ and $3 p^{2} \Sigma^{+}$, $3 p$, and $3 d^{2} \Pi$, and $3 d^{2} \Delta$, which at short $R$, coincide with the adiabatic states of CF. Based on these potentials and the ground-state potential energy curve of the cation, $\mathrm{CF}^{+}$, also calculated in the present work and listed in Table I, quantum defect functions $\eta_{i l \lambda}(R)$ have been calculated for values of $R$ between 1.8 bohr and 3.6 (for $p^{2} \Sigma^{+} 2.8$ ) bohr, in the functional form
TABLE IV. Energies ${ }^{\mathrm{a}}$ (hartree) of ns, np, and nd Rydberg states of CF at 2.2 bohr.

\begin{tabular}{rccccc}
\hline \hline$n$ & $n s^{2} \Sigma^{+}$ & $n p^{2} \Sigma^{+}$ & $n p^{2} \Pi$ & $n d^{2} \Pi$ & $n d^{2} \Delta$ \\
\hline 3 & -1.321031 & -1.279593 & -1.277336 & -1.236353 & -1.240331 \\
4 & -1.249720 & -1.236218 & -1.235495 & -1.218876 & -1.220357 \\
5 & -1.224962 & -1.218983 & -1.218660 & -1.210308 & -1.211040 \\
6 & -1.213552 & -1.210399 & -1.210227 & -1.205445 & -1.205863 \\
7 & -1.207370 & -1.205509 & -1.205406 & -1.202415 & -1.202678 \\
8 & -1.203649 & -1.202461 & -1.202394 & -1.200400 & -1.200577 \\
9 & -1.201237 & -1.200433 & -1.200387 & -1.198992 & -1.199116 \\
10 & -1.199586 & -1.199016 & -1.198983 & -1.197969 & -1.198060 \\
\hline \hline
\end{tabular}

a-136.000 000 should be added to the entries.

$$
\eta_{i}(R)=\sum_{j=0}^{4} a_{j} R^{j},
$$

and with the aid of Eq. (1). The resulting coefficients $a_{j}$ of Eq. (6) are listed in Table III. In Fig. 4, there is a plot of potential energy curves of Rydberg states of $\mathrm{CF}$ generated from the quantum defect functions while the energies of some Rydberg states at 2.2 bohr are listed in Table IV. Further information on the higher Rydberg states $n s, n p$, and $n d$ may be generated with the data provided in Table III, Table I, and Eq. (1). As it was not possible to obtain the $3 p^{2} \Sigma^{+}$ state for $R$ larger than $2.8 \mathrm{bohr}$, all the $n p^{2} \Sigma^{+}$states are limited to that distance.

\section{B. Lifetimes}

The results of some further calculations on the lower lying states of CF, $X^{2} \Pi, A^{2} \Sigma^{+}, B^{2} \Delta, 2^{2} \Pi$, and $2^{2} \Sigma^{+}$are presented below. In Fig. 5 there is a plot of the adiabatic potential energy curves of these states while in Figs. 6 and 7 there are plots of the interaction matrix elements, radial, and rotational-electronic coupling, respectively, over the electronic wavefunctions of the above states. In Fig. 8, the calculated dipole transition moments between the $X^{2} \Pi$ and the

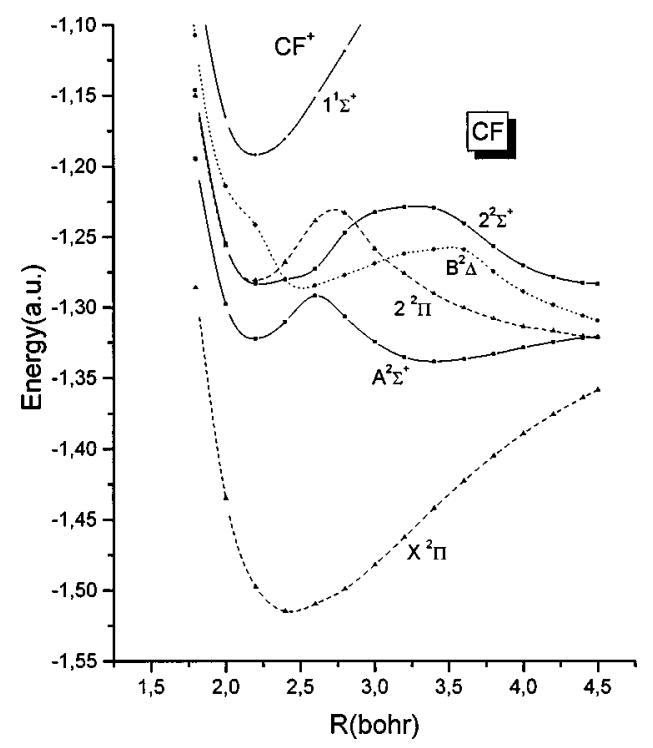

FIG. 5. Potential energy curves of the $X^{2} \Pi, A^{2} \Sigma^{+}, B^{2} \Delta, 2^{2} \Pi$, and $2^{2} \Sigma^{+}$states, involved in the CCR calculations. 


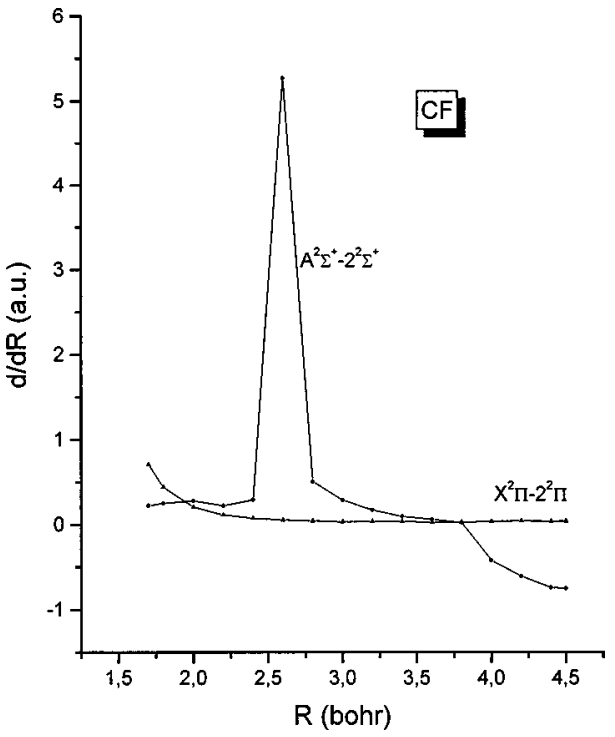

FIG. 6. Calculated radial coupling matrix elements over the electronic wavefunctions of single $C_{2 V}$ components of the states.

$A^{2} \Sigma^{+}$and $B^{2} \Delta$ states have been plotted. The dipole transition moments of the present work (see Fig. 8) are essentially identical to those of previous work ${ }^{7}$ except for the short $R$ values of the $X^{2} \Pi \rightarrow B^{2} \Delta$ transition moment, where the adiabatic $B^{2} \Delta$ state has Rydberg character because of an avoided crossing with the Rydberg $2^{2} \Delta$ state (cf Fig. 5). The calculated transition moments obtained without the Rydberg contribution, with the $B^{2} \Delta$ state following the valence curve through the crossing, and indicated by asterisks in Fig. 8 reproduce the previous results. ${ }^{7}$

The radiative lifetimes of vibrational levels of the $A^{2} \Sigma^{+}$ and $B^{2} \Delta$ states are listed in Table $\mathrm{V}$ along with experimental $(5,20)$ and previous theoretical ${ }^{7}$ values. For the $A^{2} \Sigma^{+}$state the present theoretical values should be compared with the shifted values of the previous work (values in brackets in Table V) since the present $T_{e}$ value $\left(42290 \mathrm{~cm}^{-1}\right)$ is close to

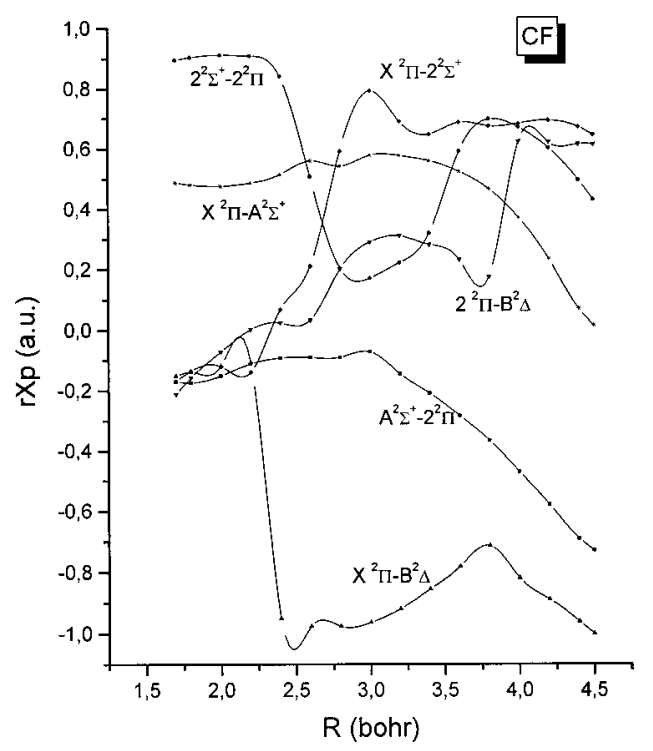

FIG. 7. Calculated rotational-electronic coupling matrix elements over the electronic wavefunctions of single $C_{2 V}$ components of the states.

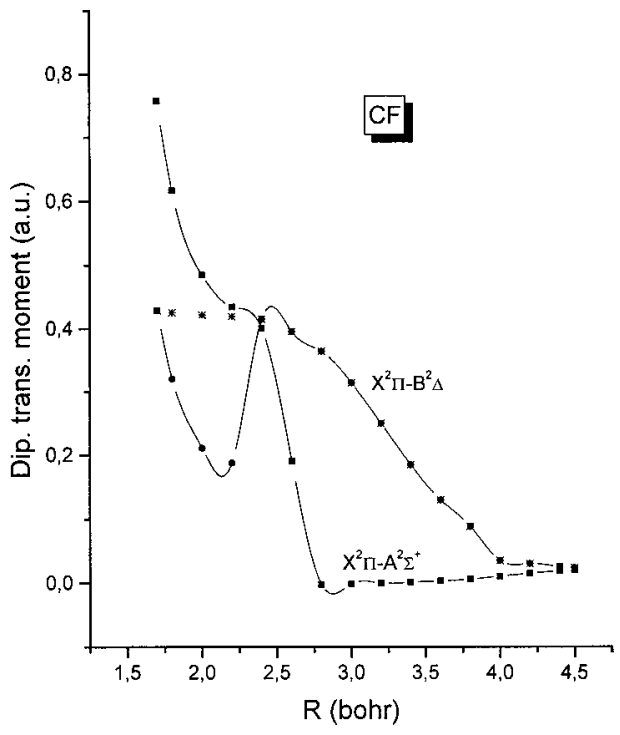

FIG. 8. Calculated dipole transition matrix elements over the electronic wavefunctions of single $C_{2 V}$ components of the states.

the experimental value $\left(42693 \mathrm{~cm}^{-1}\right)$ while for the $B^{2} \Delta$ the present values for the radiative lifetimes should be compared to the unshifted previous values since the present $T_{e}$ value (50 $564 \mathrm{~cm}^{-1}$ ) for the $X-B$ transition is closer to that of the previous theoretical value $\left(50892 \mathrm{~cm}^{-1}\right)$ than to the experimental $\left(49400 \mathrm{~cm}^{-1}\right)$. As shown in Table $\mathrm{V}$, the theoretical lifetimes are lower than the most recent experimental values for vibrational levels of $A^{2} \Sigma^{+}$(Ref. 5) and closer to earlier experimental values. ${ }^{20}$ For the $v=0$ level of the $B^{2} \Delta$ state the present work obtains a larger theoretical value for the lifetime than the previous, ${ }^{7}$ since the avoided crossing with the Rydberg state leads to lower transition moments at short $R$, and in good agreement with the available experimental data. A shift of the potential energy curve of the $B^{2} \Delta$ state to match the experimental $T_{e}$ value would make the agreement even better.

The data presented in Figs. 5, 6, and 7 have been included in a multistate complex coordinate rotation calculation of vibrational-rotational levels and their widths. These

TABLE V. Radiative lifetimes of vibrational levels of the $A^{2} \Sigma^{+}$and $B^{2} \Delta$ states of $\mathrm{CF}$.

\begin{tabular}{|c|c|c|c|c|c|c|}
\hline \multirow[t]{2}{*}{$v$} & \multicolumn{3}{|c|}{$\tau A^{2} \Sigma^{+}(\mathrm{ns})$} & \multicolumn{3}{|c|}{$\tau B^{2} \Delta(\mathrm{ns})$} \\
\hline & $\begin{array}{l}\text { Present } \\
\text { Theoret. }\end{array}$ & $\begin{array}{c}\text { Prev. } \\
\text { Theoret. }\end{array}$ & Exper. ${ }^{b, c}$ & $\begin{array}{l}\text { Present } \\
\text { Theoret. }\end{array}$ & $\begin{array}{c}\text { Prev. } \\
\text { Theoret. }\end{array}$ & Exper. $^{\mathrm{c}}$ \\
\hline 0 & 21.7 & $\begin{array}{c}23.9 \\
(22.6)\end{array}$ & $\begin{array}{l}26.7 \\
18.0 \pm 2 \\
20.0\end{array}$ & 17.7 & $\begin{array}{c}15.1 \\
(16.6)\end{array}$ & $\begin{array}{l}18.8 \pm 2, \\
19.0\end{array}$ \\
\hline 1 & 21.1 & $\begin{array}{c}23.2 \\
(22.0)\end{array}$ & $\begin{array}{l}25.6 \\
19.0 \pm 2 \\
19.0\end{array}$ & 18.0 & $\begin{array}{c}15.7 \\
(17.2)\end{array}$ & $\cdots$ \\
\hline 2 & 21.7 & $\begin{array}{c}22.9 \\
(21.7)\end{array}$ & $\cdots$ & 19.0 & $\cdots$ & $\cdots$ \\
\hline 3 & 28.7 & $\begin{array}{c}22.8 \\
(21.7)\end{array}$ & $\cdots$ & 20.0 & $\cdots$ & $\cdots$ \\
\hline
\end{tabular}

a Values in brackets obtained with shifted potential energy curves (Ref. 7).

${ }^{\mathrm{b}}$ Reference 5 .

${ }^{\mathrm{c}}$ Reference 20 . 
TABLE VI. Energy levels (hartree) ${ }^{\mathrm{a}}$ and predissociation lifetimes ${ }^{\mathrm{b}}$ resulting from the CCR vibrational calculations.

\begin{tabular}{|c|c|c|c|c|c|c|c|c|}
\hline State & $v$ & & $J=1$ & $J=2$ & $J=5$ & $J=10$ & $J=15$ & $J=20$ \\
\hline$A^{2} \Sigma^{+}$ & 0 & $\begin{array}{c}E \\
\tau\end{array}$ & $\begin{array}{c}-1.318363 \\
\ldots\end{array}$ & $\begin{array}{c}-1.318333 \\
\ldots\end{array}$ & $\begin{array}{c}-1.318151 \\
\ldots\end{array}$ & $\begin{array}{c}-1.317545 \\
\ldots\end{array}$ & $\begin{array}{c}-1.316561 \\
\ldots\end{array}$ & $\begin{array}{c}-1.315201 \\
\ldots\end{array}$ \\
\hline$A^{2} \Sigma^{+}$ & 1 & $E$ & -1.310455 & -1.310426 & -1.310245 & -1.309645 & -1.308670 & -1.307322 \\
\hline$A^{2} \Sigma^{+}$ & 2 & $\begin{array}{l}\tau \\
E \\
\tau\end{array}$ & $\begin{array}{c}-1.302786 \\
0.07 \mathrm{~ns}\end{array}$ & $\begin{array}{l}-1.302757 \\
0.07 \mathrm{~ns}\end{array}$ & $\begin{array}{c}56 \mathrm{~ns} \\
-1.302579 \\
0.07 \mathrm{~ns}\end{array}$ & $\begin{array}{c}48 \mathrm{~ns} \\
-1.301987 \\
0.06 \mathrm{~ns}\end{array}$ & $\begin{array}{c}38 \mathrm{~ns} \\
-1.301026 \\
0.05 \mathrm{~ns}\end{array}$ & $\begin{array}{c}26 \mathrm{~ns} \\
-1.299697 \\
0.04 \mathrm{~ns}\end{array}$ \\
\hline$A^{2} \Sigma^{+}$ & 3 & $\begin{array}{c}E \\
\tau\end{array}$ & $\begin{array}{c}-1.295653 \\
0.36 \mathrm{ps}\end{array}$ & $\begin{array}{c}-1.295651 \\
0.36 \mathrm{ps}\end{array}$ & $\begin{array}{c}-1.295452 \\
0.35 \mathrm{ps}\end{array}$ & $\begin{array}{c}-1.294878 \\
0.32 \mathrm{ps}\end{array}$ & $\begin{array}{c}-1.293962 \\
0.27 \mathrm{ps}\end{array}$ & $\begin{array}{c}-1.292681 \\
0.22 \mathrm{ps}\end{array}$ \\
\hline $2^{2} \Sigma^{+}$ & 0 & $\begin{array}{c}E \\
\tau\end{array}$ & $\begin{array}{c}-1.280823 \\
1.7 \mathrm{ps}\end{array}$ & $\begin{array}{c}-1.280777 \\
1.7 \mathrm{ps}\end{array}$ & $\begin{array}{c}-1.280604 \\
1.7 \mathrm{ps}\end{array}$ & $\begin{array}{c}-1.280029 \\
1.7 \mathrm{ps}\end{array}$ & $\begin{array}{c}-1.279098 \\
1.6 \mathrm{ps}\end{array}$ & $\begin{array}{c}-1.277812 \\
1.5 \mathrm{ps}\end{array}$ \\
\hline $2{ }^{2} \Pi^{+}$ & 0 & $\begin{array}{c}E \\
\tau\end{array}$ & $\begin{array}{c}-1.276799 \\
234 \mathrm{~ns}\end{array}$ & $\begin{array}{c}-1.276769 \\
70 \mathrm{~ns}\end{array}$ & $\begin{array}{c}-1.276587 \\
13 \mathrm{~ns}\end{array}$ & $\begin{array}{c}-1.275980 \\
2.4 \mathrm{~ns}\end{array}$ & $\begin{array}{c}-1.274997 \\
0.6 \mathrm{~ns}\end{array}$ & $\begin{array}{c}-1.273637 \\
0.2 \mathrm{~ns}\end{array}$ \\
\hline $2{ }^{2} \Pi^{+}$ & 1 & $\begin{array}{l}E \\
\tau\end{array}$ & $\begin{array}{c}-1.268699 \\
3.3 \mathrm{~ns}\end{array}$ & $\begin{array}{c}-1.268669 \\
1.2 \mathrm{~ns}\end{array}$ & $\begin{array}{c}-1.268488 \\
0.22 \mathrm{~ns}\end{array}$ & $\begin{array}{c}-1.267883 \\
0.06 \mathrm{~ns}\end{array}$ & $\begin{array}{c}-1.266901 \\
0.03 \mathrm{~ns}\end{array}$ & $\begin{array}{c}-1.265543 \\
0.02 \mathrm{~ns}\end{array}$ \\
\hline $2{ }^{2} \Pi^{+}$ & 2 & $\begin{array}{c}E \\
\tau\end{array}$ & $\begin{array}{c}-1.260606 \\
0.87 \mathrm{~ns}\end{array}$ & $\begin{array}{c}-1.260576 \\
0.25 \mathrm{~ns}\end{array}$ & $\begin{array}{c}-1.260396 \\
0.06 \mathrm{~ns}\end{array}$ & $\begin{array}{c}-1.259798 \\
0.02 \mathrm{~ns}\end{array}$ & $\begin{array}{c}-1.258825 \\
6.2 \mathrm{ps}\end{array}$ & $\begin{array}{c}-1.257481 \\
3.2 \mathrm{ps}\end{array}$ \\
\hline $2{ }^{2} \Pi^{+}$ & 3 & $\begin{array}{c}E \\
\tau\end{array}$ & $\begin{array}{c}-1.252619 \\
0.09 \mathrm{~ns}\end{array}$ & $\begin{array}{c}-1.252589 \\
0.03 \mathrm{~ns}\end{array}$ & $\begin{array}{c}-1.252410 \\
5.9 \mathrm{ps}\end{array}$ & $\begin{array}{c}-1.251813 \\
1.7 \mathrm{ps}\end{array}$ & $\begin{array}{c}-1.250842 \\
0.94 \mathrm{ps}\end{array}$ & $\begin{array}{c}-1.249499 \\
0.74 \mathrm{ps}\end{array}$ \\
\hline$B^{2} \Delta^{+}$ & 0 & $\begin{array}{c}E \\
\tau\end{array}$ & & $\begin{array}{c}-1.282874 \\
\ldots\end{array}$ & $\begin{array}{c}-1.282736 \\
\ldots\end{array}$ & $\begin{array}{c}-1.282275 \\
\ldots\end{array}$ & $\begin{array}{c}-1.281527 \\
\ldots\end{array}$ & $\begin{array}{c}1.280492 \\
\ldots\end{array}$ \\
\hline$B^{2} \Delta^{+}$ & 1 & $\begin{array}{c}E \\
\tau\end{array}$ & & $\begin{array}{c}-1.277302 \\
\ldots\end{array}$ & $\begin{array}{c}-1.277171 \\
\ldots\end{array}$ & $\begin{array}{c}-1.276734 \\
\ldots\end{array}$ & $\begin{array}{c}-1.276025 \\
\ldots\end{array}$ & $\begin{array}{c}1.275045 \\
7 \mu \mathrm{s}\end{array}$ \\
\hline$B^{2} \Delta^{+}$ & 2 & $\begin{array}{c}E \\
\tau\end{array}$ & & $\begin{array}{c}-1.272491 \\
1.2 \mu \mathrm{s}\end{array}$ & $\begin{array}{c}-1.272365 \\
700 \mathrm{~ns}\end{array}$ & $\begin{array}{c}-1.271946 \\
160 \mathrm{~ns}\end{array}$ & $\begin{array}{c}-1.271264 \\
63 \mathrm{~ns}\end{array}$ & $\begin{array}{c}-1.270322 \\
29 \mathrm{~ns}\end{array}$ \\
\hline$B^{2} \Delta^{+}$ & 3 & $\begin{array}{c}E \\
\tau\end{array}$ & & $\begin{array}{c}-1.268103 \\
5 \mathrm{~ns}\end{array}$ & $\begin{array}{c}-1.267982 \\
15 \mathrm{~ns}\end{array}$ & $\begin{array}{c}-1.267577 \\
3.6 \mathrm{~ns}\end{array}$ & $\begin{array}{c}-1.266921 \\
1.3 \mathrm{~ns}\end{array}$ & $\begin{array}{l}1.266015 \\
0.76 \mathrm{~ns}\end{array}$ \\
\hline$B^{2} \Delta^{+}$ & 4 & $\begin{array}{c}E \\
\tau\end{array}$ & & $\begin{array}{c}-1.264110 \\
0.04 \mathrm{~ns}\end{array}$ & $\begin{array}{c}-1.263993 \\
1.4 \mathrm{~ns}\end{array}$ & $\begin{array}{c}-1.263606 \\
0.48 \mathrm{~ns}\end{array}$ & $\begin{array}{c}-1.262977 \\
0.22 \mathrm{~ns}\end{array}$ & $\begin{array}{c}-1.262109 \\
0.13 \mathrm{~ns}\end{array}$ \\
\hline
\end{tabular}

a -136.000000 should be added to the energy entries.

${ }^{\mathrm{b}}$ For predissociation lifetimes shorter than $10 \mu \mathrm{s}$.

calculations concern only the positive parity substates of the ${ }^{2} \Pi$ and ${ }^{2} \Delta$ states along with the ${ }^{2} \Sigma^{+}$states. ${ }^{21}$ The results are summarized in Table VI in terms of energies of different vibrational-rotational levels and the predissociation lifetimes. It is possible to assign the vibrational-rotational levels to particular electronic states on the basis of the dominant coefficient. Only lifetimes shorter than $10 \times 10^{-6}$ seconds are given. As shown in Table VI, the present results on the levels of the $A^{2} \Sigma^{+}$state are in agreement with previous results and observations. The levels $v=0$ and $v=1$ are not predissociated and only for rotational quantum number $J$ of 20 is the predissociative lifetime of the $v=1$ level close to the value of the radiative lifetime. For higher vibrational levels very short predissociation lifetimes are obtained (see Table VI). For $v=2$, the predissociation lifetime is $0.07 \mathrm{~ns}$ for $J=1$ and decreases only slightly with $J$ giving $0.04 \mathrm{~ns}$ for $J=20$. The experimentally determined range for the predissociation lifetime is from 0.4 to $2 \mathrm{~ns}$, while the tunneling lifetime for rotationless $v=2$ is calculated at $4.2 \mathrm{~ns}$ (Ref. 7) and for different rotational levels ${ }^{5}$ it varies from $3 \mathrm{~ns}$ (for $J=0$ ) to 2 ns (for $J=20$ ). For $v=3$, the predissociation lifetime is 0.36 to 0.22 ps (between $J=1$ and $J=20$ ) with the tunneling lifetime 4.1 ps. $^{7}$ Thus the present results are in agreement with experimental observations regarding the predissociation of the different vibrational levels and regarding the slow variation of the lifetimes with rotational level. The absolute values of the lifetimes obtained in the present work are a factor of 10 lower than the experimentally estimated lower bound. ${ }^{5}$

The predissociation lifetimes of the $2^{2} \Sigma^{+}$state show that it is heavily predissociated with lifetimes of the order of picoseconds (see Table VI). The $2^{2} \Sigma^{+}$state lies in the vibrational continuum of the $A^{2} \Sigma^{+}$and it has very strong radial coupling with it (cf. Figs. 5 and 6) and in this manner it is predissociated. The assigned band at $6.35 \mathrm{eV}$ to the $X^{2} \Pi \rightarrow 3 p 2^{2} \Sigma^{+}$transition is reported as diffuse, ${ }^{6}$ which is consistent with short predissociation lifetimes for this state. The $D^{2} \Pi$ state (or $2^{2} \Pi$ ) is not strongly predissociated for rotational levels $J<5$ of $v=0$, while predissociation becomes important for higher vibrational levels. The mechanism seems to be via rotational-electronic coupling, since there is strong decrease of the lifetime with $J$ (see Table VI). As shown in Fig. 7, the largest interaction of the $D^{2} \Pi$ state is with $2^{2} \Sigma^{+}$and therefore it is predissociated indirectly by $A^{2} \Sigma^{+}$. The results for levels of the $B^{2} \Delta$ state (see Table VI) show that the vibrational levels $v=0$ and $v=1$ are not significantly predissociated but for higher $v$ levels predissociation is faster than the radiative $B^{2} \Delta-X^{2} \Pi$ transition. Again, the predissociation lifetime decreases with increasing $J$, consistent with the strong rotational-electronic coupling of this state with the ground state, $X^{2} \Pi$. Thus only the levels of the positive parity substate of $D^{2} \Pi$ are expected to be affected while both the levels of positive and the negative parity substates of $B^{2} \Delta$ are predicted to be equally predissociated.

\section{CONCLUSION}

In the present work $a b$ initio MRDCI calculations are presented on several electronic states of $\mathrm{CF}$ and on the 
ground state of $\mathrm{CF}^{+}$. An effort has been made to rationalize the complicated shapes of the potential energy curves in terms of Rydberg and valence contributions and quantum defect functions have been determined which have been employed in order to generate higher-lying Rydberg states of $\mathrm{CF}$. On the basis of the electronic transition energies the observed bands involving the $F, G$, and $H$ states of $C F$ have been assigned to the $3 d 2^{2} \Delta, 3^{2} \Pi$, and $4^{2} \Sigma^{+}$states, respectively, while additional observed bands may be associated with some of the other electronic states calculated in the present work. Radiative lifetimes have been calculated for vibrational levels of the $A^{2} \Sigma^{+}$and $B^{2} \Delta$ states showing that better agreement with experiment is obtained when the valence-Rydberg interaction of the $B^{2} \Delta$ at short $R$ is taken into account. Complex coordinate vibrational calculations over the $X^{2} \Pi, A^{2} \Sigma^{+}, B^{2} \Delta, 2^{2} \Pi$, and $2^{2} \Sigma^{+}$states have obtained predissociation widths and associated lifetimes for vibrational-rotational levels of these states. In agreement with experiment, short predissociation lifetimes are calculated for levels $v>1$ of the $A^{2} \Sigma^{+}$state. The $2^{2} \Sigma^{+}$state is found to be heavily predissociated even for $v=0$, while $J$-dependent predissociation of a smaller magnitude is obtained for the states $B^{2} \Delta$ and $2^{2} \Pi$.

${ }^{1}$ E. B. Andrews and R. F. Barrow, Proc. Phys. Soc. London, Sect. A 64, 481 (1951); T. L. Porter, D. E. Mann, and N. Acquista, J. Mol. Spectrosc. 16, 228 (1965).

${ }^{2}$ B. A. Thrush and J. J. Zwolenik, Trans. Faraday Soc. 59, 582 (1963).

${ }^{3}$ P. K. Carroll and T. P. Grennan, J. Phys. B 3, 865 (1970).

${ }^{4}$ F. J. Grieman, A. T. Droege, and P. C. Engelking, J. Chem. Phys. 78, 2248 (1983)
${ }^{5}$ J. P. Booth, G. Hancock, M. J. Toogood, and K. G. McKendrick, J. Phys. Chem. 100, 47 (1996); J. P. Booth and G. Hancock, Chem. Phys. Lett. 150, 457 (1988).

${ }^{6}$ T. H. Dunning, Jr., W. P. White, R. M. Pitzer, and C. W. Mathews J. Mol. Spectrosc. 75, 297 (1979); W. P. White, R. M. Pitzer, C. W. Mathews, and T. H. Dunning, Jr., ibid. 75, 318 (1979).

${ }^{7}$ A. P. Rendell, C. W. Bauschlicher, Jr., and S. R. Langhoff, Chem. Phys. Lett. 163, 354 (1989).

${ }^{8}$ R. J. Buenker, in Studies in Physical and Theoretical Chemistry, Current Aspects of Quantum Chemistry edited by R. Carbo (Elsevier, Amsterdam, 1981) Vol. 2, p. 17; R. I. Buenker and R. A. Phillips, J. Mol. Struct.: THEOCHEM 123, 291 (1985).

${ }^{9}$ T. H. Dunning, J. Chem. Phys. 55, 716 (1971).

${ }^{10}$ T. H. Dunning and J. P. Hay, in Methods in Electronic Structure Theory, edited by H. F. Schaefer III (Plenum Press, New York, 1977), p. 1.

${ }^{11}$ S. R. Langhoff and E. R. Davidson, Int. J. Quantum Chem. 8, 61 (1974).

${ }^{12}$ I. D. Petsalakis, G. Theodorakopoulos, Y. Li, G. Hirsch, R. J. Buenker, and M. S. Child, J. Chem. Phys. 108, 7607 (1998).

${ }^{13}$ C. H. Greene and Ch. Jungen, Adv. At. Mol. Phys. 21, 51 (1985); S. C. Ross and Ch. Jungen, Phys. Rev. A 49, 4353 (1994); 49, 4364 (1994); M. Seaton, Rep. Prog. Phys. 46, 167 (1983).

${ }^{14}$ I. D. Petsalakis, R. J. Buenker, G. Hirsch, and G. Theodorakopoulos, J. Phys. B. At. Mol. Opt. Phys. 30, 4935 (1997).

${ }^{15}$ K. P. Huber and G. Herzberg, Constants of diatomic molecules (Van Nostrand Reinhold, New York, 1979).

${ }^{16}$ T. A. Walter, C. Lifshitz, W. A. Chupka, and J. Berkowitz, J. Chem. Phys. 51, 3531 (1969).

${ }^{17}$ D. L. Hildenbrand, Chem. Phys. Lett. 32, 523 (1975).

${ }^{18}$ J. M. Dyke, A. E. Lewis, and A. Morris, J. Chem. Phys. 80, 1382 (1984).

${ }^{19}$ J. W. Hepburn, D. J. Trevor, J. E. Pollard, D. A. Shirley, and Y. T. Lee, J. Chem. Phys. 76, 4287 (1982).

${ }^{20}$ H. A. van Sprang, H. H. Brongersma, and F. DeHeer, Chem. Phys. 35, 51 (1978); J. E. Hesser, J. Chem. Phys. 48, 2518 (1968); T. Wentink and L. Isaacson, ibid. 46, 603 (1967); J. Harrington, A. P. Modica, and D. R. Libby, ibid. 44, 3380 (1966).

${ }^{21}$ G. Herzberg, Molecular Spectra and Molecular Structure I. Spectra of Diatomic Molecules (Van Nostrand Reinhold, New York, 1950), p. 417. 